\title{
Thermal decomposition synthesis of functionalized PdPt alloy nanodendrites with high selectivity for oxygen reduction reaction
}

\author{
Fu-Min $\mathrm{Li}^{1}$, Xue-Qing Gao ${ }^{1}$, Shu-Ni Li ${ }^{1}$, Yu Chen ${ }^{1}$ and Jong-Min $\mathrm{Lee}^{2}$
}

Pt-based bimetallic nanostructures have found intriguing applications in electrocatalysis. However, the pristine Pt-based nanostructures generally lack the selectivity for the target reaction because of their high activity for both oxygen reduction reactions (ORRs) and fuel molecule oxidation reactions. By employing a recently developed chemical functionalization strategy, the functionalized Pt-based nanostructures have achieved their selectivity for the target reaction in fuel cells. In this work, we report a facile thermal decomposition route to synthesize the polyallylamine (PAH)-functionalized Pd-Pt bimetallic core-shell nanodendrites with a Pd-rich PdPt alloy core and a Pt-rich PtPd alloy shell (PdPt@PtPd CSNDs) by using PAH that serves as a complexant, reductant and chemical functionalization molecule. The composition, morphology and structure of PdPt@PtPd CSNDs are characterized in detail. Compared with commercial Pt black electrocatalyst, the PAH-functionalized PdPt@PtPd CSNDs show improved electrocatalytic activity and durability for the ORR, and achieve good selectivity for the ORR in the presence of ethanol molecules. The study shows a promising cathode electrocatalyst for direct alcohol fuel cells (DAFCs). NPG Asia Materials (2015) 7, e219; doi:10.1038/am.2015.108; published online 16 October 2015

\section{INTRODUCTION}

Direct alcohol fuel cells (DAFCs), an electrocatalytic energy conversion device that can directly convert the chemical energy of alcohol molecules (such as methanol, ethanol, propanol and so on) into electricity, is a highly promising power source in transportation and portable electronics. ${ }^{1-5}$ Nowadays, one of the key challenges in the DAFC commercialization is to improve the efficiency, activity, alcohol tolerance and durability of cathodic Pt electrocatalysts for the oxygen reduction reaction (ORR). ${ }^{1,2,6,7}$ So far, many advanced Pt-based nanostructures with high ORR performance have been achieved by controlling their crystal facets, ${ }^{8,9}$ composition, ${ }^{10-15}$ architecture ${ }^{16,17}$ and interface structure. ${ }^{18,19}$ Among various Pt-based nanostructures, three-dimensionally (3D) interconnected Pt-Pd bimetallic nanostructures show superior ORR activity and durability owing to their fascinating physical and chemical properties, such as (1) the superior electrochemical self-stability that effectively restrain dissolution under electrochemical conditions, owing to the high stability of the $\mathrm{Pd}$ element in acidic electrolyte; (2) the big surface area accessible to the reactant molecules; and (3) the particular 3D self-supported architecture that effectively restrain the Ostwald ripening effect, improve the electron transport in the solid ligaments and facilitate the mass transfer of oxygen molecules. ${ }^{20-23}$
Apart from the activity and durability, the selectivity (that is, ethanol tolerance) is also a key issue for the synthesis of highperformance cathodic Pt electrocatalysts because the alcohol crossover always happens in DAFCs. In recent pioneering studies, ${ }^{24-26}$ Marković and co-workers ${ }^{24,25}$ found that calix[4]arene molecule modified $\mathrm{Pt}$ could selectively block the ORR without affecting the hydrogen oxidation reaction activity and kinetics. Meanwhile, Yang andcolleagues ${ }^{26}$ found cage-bell structured Pt-Ru nanoparticles could selectively block the methanol oxidation reaction but allow the ORR because of the difference of molecular size between the methanol and oxygen molecules. Since then, some chemical functionalized $\mathrm{Pt}$ nanocrystals have successfully achieved the selectivity for the ORR in the presence of alcohol molecules because of the strong ensemble effects. ${ }^{6,19}$ For example, in our previous work, we synthesized the polyallylamine (PAH, Supplementary Scheme S1)-functionalized Pt nanolances by chemical reduction of $\mathrm{PAH}-\mathrm{Pt}^{\mathrm{II}}$ complex using $\mathrm{HCHO}$ that exhibited a high ORR selectivity in the presence of methanol. ${ }^{19}$ However, the as-prepared Pt nanolances suffered from harsh and tedious ultraviolet (UV)/ozone post-treatment procedures before electrocatalytic applications. In this work, we report a one-step water-based thermal decomposition route to synthesize the PAH-functionalized Pd-Pt bimetallic core-shell nanodendrites with

\footnotetext{
${ }^{1}$ Key Laboratory of Macromolecular Science of Shaanxi Province, School of Materials Science and Engineering, School of Chemistry and Chemical Engineering, Shaanxi Normal University, Xi'an, People's Republic of China and ${ }^{2}$ School of Chemical and Biomedical Engineering, Nanyang Technological University, Singapore

Correspondence: Professor Y Chen, Key Laboratory of Macromolecular Science of Shaanxi Province, School of Materials Science and Engineering, Shaanxi Normal University, Xi'an 710062, People's Republic of China.

E-mail: ndchenyu@gmail.com

or Professor J-M Lee, School of Chemical and Biomedical Engineering, Nanyang Technological University, Singapore 637459, Singapore.

E-mail: jmlee@ntu.edu.sg
}

Received 14 April 2015; revised 23 July 2015; accepted 28 July 2015 
a Pd-rich PdPt alloy core and a Pt-rich PtPd alloy shell (PdPt@PtPd CSNDs) under hydrothermal conditions by using PAH that serves as a complexant, reductant and chemical functionalization molecule that can directly be used as a cathodic ORR electrocatalyst without tedious UV/ozone post-treatment procedure. The as-synthesized PAH-functionalized PdPt@PtPd CSNDs have high activity, good durability and fascinating selectivity for the ORR.

\section{EXPERIMENTAL PROCEDURE}

\section{Reagents and chemicals}

PAH (Supplementary Scheme S1, average molecular weight 150000) was supplied from Nitto Boseki Co., Ltd (Tokyo, Japan). Potassium tetrachloroplatinate(II) $\left(\mathrm{K}_{2} \mathrm{PtCl}_{4}\right)$ and potassium tetrachloropalladate(II) $\left(\mathrm{K}_{2} \mathrm{PdCl}_{4}\right)$ were purchased from Sinopharm Chemical Reagent Co., Ltd (Shanghai, China). Commercial Pt black was purchased from Johnson Matthey Corporation (London, UK). Other reagents were of analytical reagent grade and used without further purification.

\section{Preparation of PdPt@PtPd CSND}

In a typical synthesis, $1 \mathrm{ml}$ of $0.025 \mathrm{M} \mathrm{K}_{2} \mathrm{PtCl}_{4}, 1.1 \mathrm{ml}$ of $0.025 \mathrm{M} \mathrm{K}_{2} \mathrm{PdCl}_{4}$ and $0.6 \mathrm{ml}$ of $0.50 \mathrm{M}$ PAH $(\mathrm{Mw}=150000$, molarity of $\mathrm{PAH}$ given with respect to the repeating unit) were added into $7.0 \mathrm{ml}$ water under stirring. Then, the mixture solution was transferred into a Teflon-lined stainless-steel autoclave and heated at $180^{\circ} \mathrm{C}$ for $6 \mathrm{~h}$. After the recation, the products were collected by centrifugation and further washed five times with water.

\section{Electrochemical measurements}

All electrochemical experiments were carried out on a CHI $660 \mathrm{C}$ electrochemical workstation ( $\mathrm{CH}$ instruments, Inc. Shanghai, China) at $30 \pm 1{ }^{\circ} \mathrm{C}$, using a standard three-electrode system. Rotating disk electrode (RDE) measurements were performed on a RDE710 Gamry RDE (Gamry Instruments, Warminster, PA, USA). A Pt wire and a saturated calomel electrode protected by Luggin capillary with a $\mathrm{KCl}$ solution were used as counter and reference electrodes, respectively. Potentials in this study were given relative to the reversible hydrogen electrode. The working electrode was prepared by mixing the electrocatalyst $(10 \mathrm{mg}$ ) and the isopropanol/Nafion (Sigma Corporation, Cream Ridge, NJ, USA) solution (20\% isopropanol and $0.02 \%$ Nafion), and sonicating the solution for $60 \mathrm{~min} .^{27}$ Then, $10 \mu \mathrm{l}$ of the catalyst ink was put onto a clean glassy carbon electrode $\left(5 \mathrm{~mm}\right.$ diameter, $\left.0.196 \mathrm{~cm}^{2}\right)$ and dried at room temperature. The specific loading of the metal on the electrode surface was about $102 \mu \mathrm{g} \mathrm{cm}^{-2}$

\section{Characterization}

Morphology, bulk composition, surface structure and crystal structure of PdPt@PtPd CSNDs were characterized by scanning electron microscopy (JSM-2010, JEOL, Tokyo, Japan) with energy-dispersive X-ray (EDX) accessory, transmission electron microscopy (TEM, JEM-2100F, JEOL), inductively coupled plasma atomic emission spectrometry (Leeman Labs, Inc. Hudson, $\mathrm{NH}$, USA) and X-ray diffraction (Model D/max-rC, Rigaku Corporation, Tokyo, Japan). The surface composition of PdPt@PtPd CSNDs was investigated by X-ray photoelectron spectroscopy (Thermo VG Scientific, Waltham, MA, USA, ESCALAB 250). The binding energy was calibrated by means of the $\mathrm{C}$ 1s peak energy of $284.6 \mathrm{eV}$. Thermogravimetric analysis was performed on a thermogravimetric analyzer (TA Q600SDT, TA instruments, Inc. New Castle, DE, USA) under $\mathrm{N}_{2}$ atmosphere. After the reaction, molecular weight of PAH was calculated by measuring the intrinsic viscosity of PAH solution ${ }^{28,29}$ using AMVn viscometer (Anton Paar, Graz, Austria).

\section{RESULTS AND DISCUSSION}

The preparation process of PAH-functionalized PdPt@PtPd CSNDs is illustrated in Scheme 1. In a typical synthesis, PAH-functionalized PdPt@PtPd CSNDs are produced by heating an aqueous solution containing $\mathrm{K}_{2} \mathrm{PtCl}_{4}, \mathrm{~K}_{2} \mathrm{PdCl}_{4}$ and $\mathrm{PAH}$ for $6 \mathrm{~h}$ at $180{ }^{\circ} \mathrm{C}$ (see Experimental procedures section for details). Herein, $\mathrm{PAH}$ first reacts

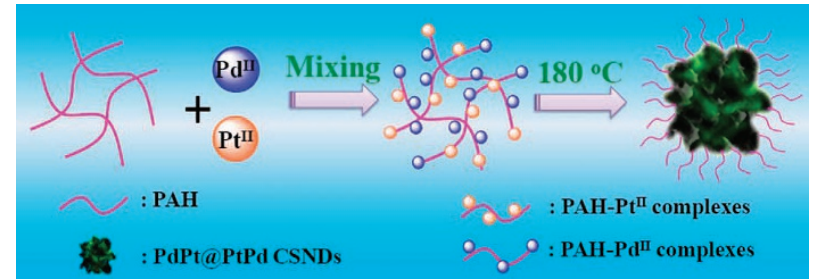

Scheme 1 Schematic synthetic route of polyallylamine (PAH)-functionalized PdPt@PtPd CSNDs by the facile thermal decomposition route.

with $\mathrm{K}_{2} \mathrm{PtCl}_{4}$ and $\mathrm{K}_{2} \mathrm{PdCl}_{4}$ to generate $\mathrm{PAH}-\mathrm{Pt}{ }^{\mathrm{II}}\left(\mathrm{Fu}\right.$ et al. ${ }^{30}$ ) and $\mathrm{PAH}-\mathrm{Pd}{ }^{\mathrm{II}}$ (Fu et al. ${ }^{31}$ ) complexes at room temperature, respectively, owing to the strong coordinating capability of PAH. Then, $\mathrm{PAH}-$ functionalized PdPt@PtPd CSNDs were obtained by simultaneous thermal decomposition of both PAH-Pt ${ }^{\mathrm{II}}$ and PAH-Pd ${ }^{\mathrm{II}}$ complexes at high temperatures. During the thermal decomposition, the long carbon chain of PAH breaks up, resulting in the generation of $\mathrm{PAH}$ with a small molecular weight $(\mathrm{Mw}=14000)$. Simultaneously, $\mathrm{PAH}$ with a small molecular weight firmly binds on the PdPt@PtPd CSNDs surface because of interaction between $-\mathrm{NH}_{2}$ groups and metal atoms. ${ }^{30,31}$ The PAH-functionalized PdPt@PtPd CSNDs can be used as a cathodic ORR electrocatalyst after centrifugation, washing and drying, without further tedious UV/ozone post-treatment procedure of the electrocatalysts.

EDX, inductively coupled plasma atomic emission spectrometry and X-ray diffraction measurements were carried out to characterize the bulk composition and crystal structure of PdPt@PtPd CSNDs. On the basis of EDX analysis (Supplementary Figure S1), the Pd-Pt atomic ratio of prodcuts is measured to be $53: 47$, close to inductively coupled plasma atomic emission spectrometry analysis (56:44). Compared with standard diffraction data of single Pt (JCPDS-04$0802 \mathrm{Pt}$ ) and Pd (JCPDS-46-1043Pd), the representative diffraction peaks of PdPt@PtPd CSNDs are coincidently located between them (Figure 1a), demonstrating the formation of the solid-solution Pt-Pd alloy. The crystalline size of PdPt@PtPd CSNDs is estimated to be $5 \mathrm{~nm}$ according to the Scherrer formula. X-ray photoelectron spectroscopy was performed to investigate the surface composition and electronic structure of PdPt@PtPd CSNDs. X-ray photoelectron spectroscopy measurement shows the $\mathrm{Pd} / \mathrm{Pt}$ atomic ratio is 39:61 (Supplementary Figure S2), much lower than that measured by inductively coupled plasma atomic emission spectrometry $(\mathrm{Pd} / \mathrm{Pt}=$ 56:44), hinting at the formation of a Pt-rich PtPd alloy shell. The detailed X-ray photoelectron spectroscopy analyses demonstrate the metallic Pt and Pd are predominant in PdPt@PtPd CSNDs (Figures 1b and $\mathrm{c}$ ), revealing the complete reduction of $\mathrm{Pt}^{\mathrm{II}}$ and $\mathrm{Pd}^{\mathrm{II}}$ precursors. Compared with the standard values of bulk $\mathrm{Pt}$ and $\mathrm{Pd},{ }^{32}$ the binding energies of Pt 4f and Pd 3d in PdPt@PtPd CSNDs negetively shift 0.7 and $0.4 \mathrm{eV}$, respectively. Meanwhile, $\mathrm{N}$ 1s signal is detected (Figure 1d), indicating PAH binds on the PdPt@PtPd CSNDs surface. Because of the small discrepancy between electronegativity of $\mathrm{Pt}$ and Pd (Pt: 2.28 vs Pd: 2.20), the change in the binding energies of Pt $4 \mathrm{f}$ and $\mathrm{Pd} 3 \mathrm{~d}$ mainly orignates from the electron donation induced by $-\mathrm{NH}_{2}$ groups in the $\mathrm{PAH}$ with the lone pair of electrons ${ }^{19}$ rather than the interaction between Pt and Pd elements.

The morphology and surface structure of PdPt@PtPd CSNDs were further investigated by scanning electron microscopy and TEM. The scanning electron microscopy image clearly reveals that PdPt@PtPd CSNDs have a rough surface (Figure 2a). The TEM images show most of PdPt@PtPd CSNDs have a dendritic shape and a narrow size 
a

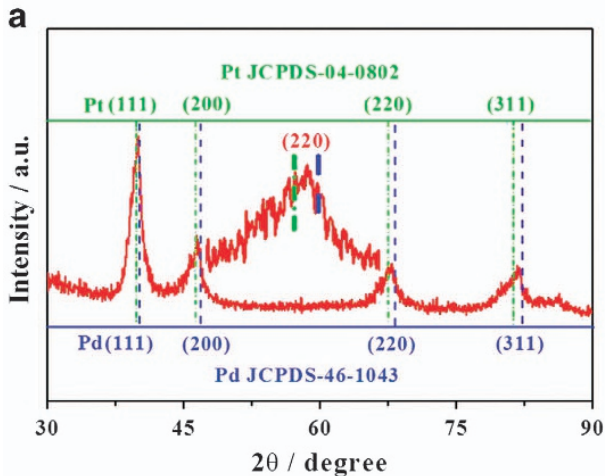

C

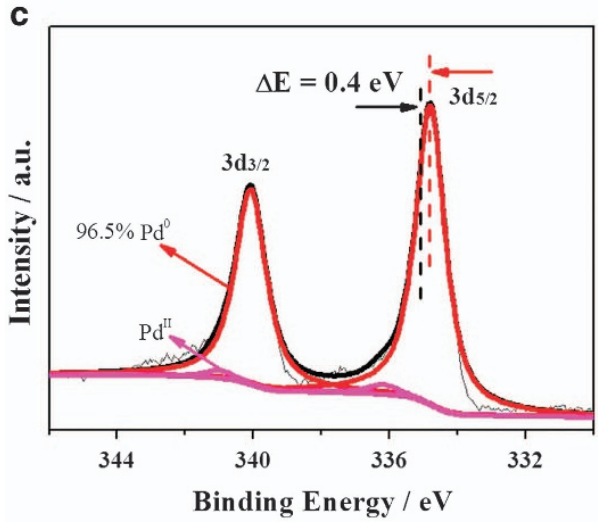

b

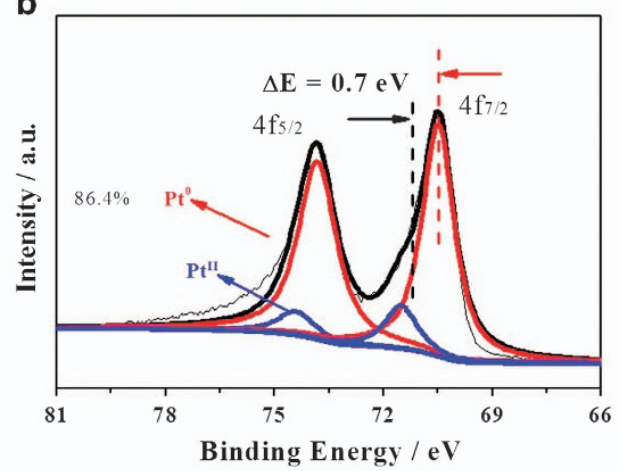

d

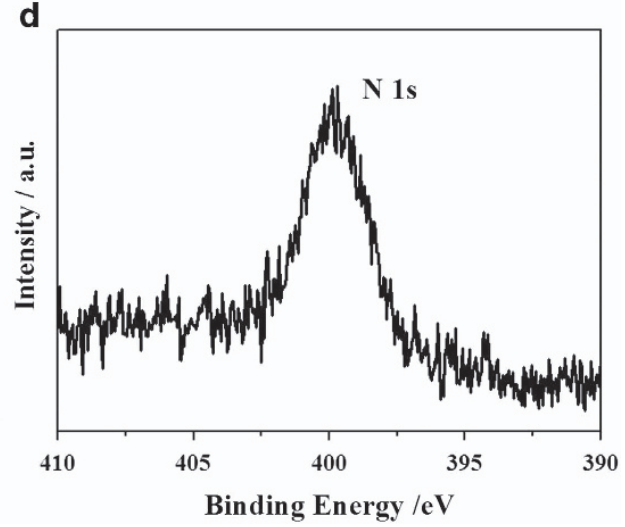

Figure 1 (a) X-ray diffraction (XRD) pattern, (b) Pt 4f X-ray photoelectron spectroscopy (XPS) spectrum, (c) Pd 3d XPS spectrum and (d) N 1s XPS spectrum of PdPt@PtPd CSNDs. (Note that the vertical black dotted lines in $\mathbf{b}$ and $\mathbf{c}$ represent the standard value of Pt 4f7/2 and Pd 3d5/2, respectively.).

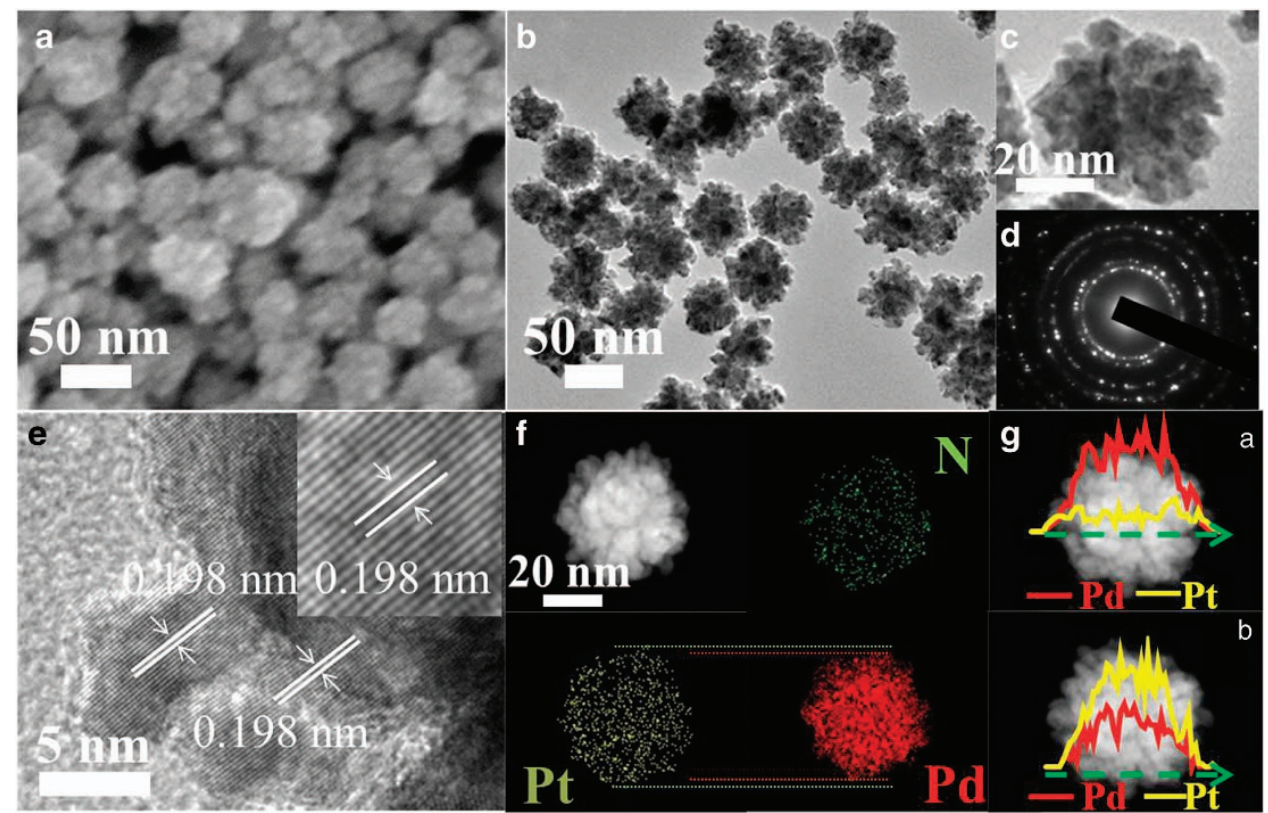

Figure 2 (a) Scanning electron microscopy (SEM), (b, c) transmission electron microscopy (TEM), (d) selected area electron diffraction (SAED) and (e) highresolution TEM (HRTEM) images of PdPt@PtPd CSNDs. (f) High-angle annular dark-field scanning TEM and EDX mapping images of N, Pt and Pd elements. (g) Line scanning profiles of PdPt@PtPd CSNDs on (a) center and (b) edge of individual particle.

distribution of $42-48 \mathrm{~nm}$ (Figure 2b) that consist of many subunit nanoparticles with ca. $6 \mathrm{~nm}$ diameters (Figure 2c). The selected area electron diffraction image shows the irregular spot pattern (Figure 2d), indicating their polycrystalline nature.
The high-resolution TEM image taken from several subunit nanoparticles clearly shows well-resolved fringes with lattice spacing of $0.198 \mathrm{~nm}$ (Figure 2e) that is indexed to $\{100\}$ crystal facets of facecentered cubic Pd-Pt alloy. At $140^{\circ} \mathrm{C}, \mathrm{K}_{2} \mathrm{PtCl}_{4}$ precursors could not be 
reduced by PAH (Supplementary Figure S3). At $220^{\circ} \mathrm{C}$, the PtPd nanoparticles generated severe aggregation (Supplementary Figure S4). Consequently, $180^{\circ} \mathrm{C}$ was selected as the optimal synthesis temperature.

EDX elemental analysis mapping and EDX line scanning were performed to investigate the surface composition and the positional distribution of the Pd, Pt and N elements in PdPt@PtPd CSNDs. The pattern of the $\mathrm{N}$ element is very similar to the patterns of the Pd and Pt elements (Figure 2f), demonstrating the uniform binding of PAH on the PdPt@PtPd CSNDs surface because of strong N-Pt and N-Pd bond interactions. ${ }^{30,31}$ Meanwhile, the diameter of the Pt element pattern is slightly bigger than that of the $\mathrm{Pd}$ element pattern, confirming the core-shell structure of PdPt@PtPd CSNDs (Figure 2f). EDX line scanning spectrum on the center of the individual PdPt@PtPd CSNDs shows the presence of Pt and Pd elements (Figure 2ga), and the content of the Pd element is much higher than that of the Pt element (Figure 2ga). In contrast, the content of the Pt element is higher than that of the Pd element on the edge of the individual PdPt@PtPd CSNDs (Figure 2gb). Thus, EDX line scanning spectra on the two positions clearly confirm that
PdPt@PtPd CSNDs have a Pd-rich PdPt alloy core and a Pt-rich PtPd alloy shell. TEM images show that the single-component Pd nanodendrites and Pt nanodendrites can also be synthesized using the same thermal decomposition method (Supplementary Figure S5). Thermogravimetric analysis tests show the decomposition temperature of the PAH-Pd ${ }^{\mathrm{II}}$ complex is lower than that of the PAH-Pt ${ }^{\mathrm{II}}$ complex (Supplementary Figure S6). Meanwhile, linear sweeping voltammetry measurements show the onset reduction potential of $\mathrm{PAH}-\mathrm{Pd}^{\mathrm{II}}$ complex is higher than that of PAH-Pt ${ }^{\mathrm{II}}$ complex (Supplementary Figure S7). Real time-dependent photographs show the onset conversion of PAH-Pd ${ }^{\mathrm{II}}$ complex and $\mathrm{PAH}-\mathrm{Pt}^{\mathrm{II}}$ complex to $\mathrm{Pd}$ nanoparticles and Pt nanoparticles occur at 0.5 and $1 \mathrm{~h}$, respectively (Supplementary Figure S8). Thus, the preferential reduction of the $\mathrm{Pd}^{\mathrm{II}}$ precursor plays a key role in the formation of the particular core-shell nanostructures with a Pd-rich PdPt alloy core and a Pt-rich PtPd alloy shell.

The electrochemical behavior of PdPt@PtPd CSNDs in a $0.5 \mathrm{M}$ $\mathrm{H}_{2} \mathrm{SO}_{4}$ solution was studied by cyclic voltammetry $(\mathrm{CV})$ and compared with that of commercial Pt black (Figure 3a). No detectable reduction peak of Pd oxide is observed in cyclic voltammogram for PdPt@PtPd CSNDs, confirming the alloy nature of the Pt-Pd shell.
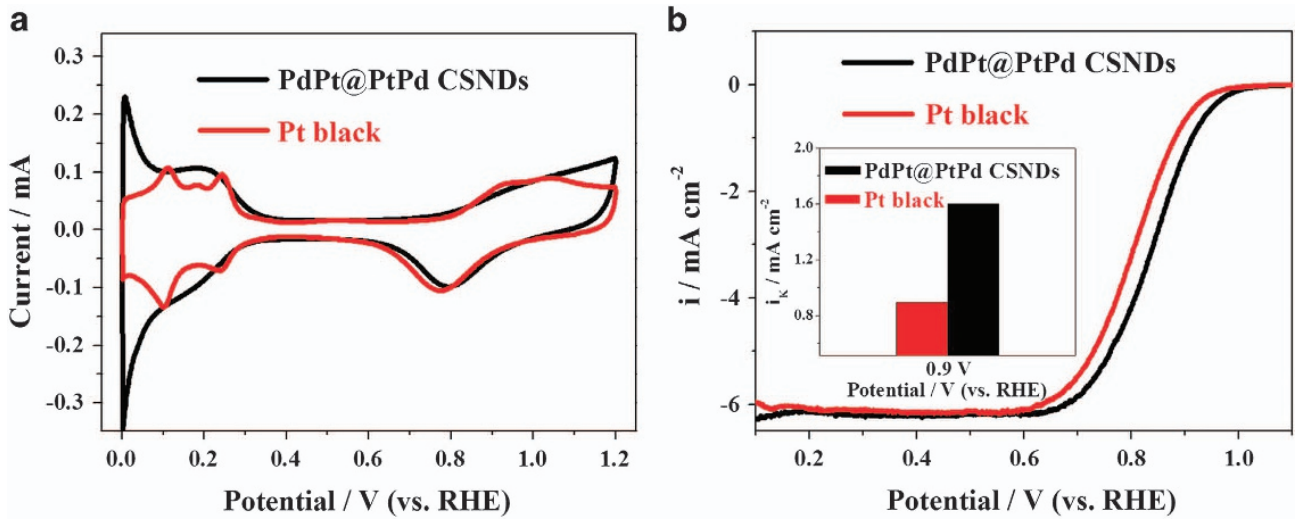

Figure 3 (a) Cyclic voltammograms of PdPt@PtPd CSNDs and Pt black in $\mathrm{N}_{2}$-saturated $0.5 \mathrm{M} \mathrm{H}_{2} \mathrm{SO}_{4}$ electrolyte at $50 \mathrm{mV} \mathrm{s}^{-1}$. (b) Oxygen reduction reaction (ORR) polarization curves of PdPt@PtPd CSNDs and Pt black in $\mathrm{O}_{2}$-saturated $0.5 \mathrm{M} \mathrm{H}_{2} \mathrm{SO}_{4}$ electrolyte at $5 \mathrm{mV} \mathrm{s}^{-1}$ and rotation rate of 1600 r.p.m. Insert indicates the $i_{k}$ values of PdPt@PtPd CSNDs and Pt black at a fixed potential of $0.9 \mathrm{~V}$ for the ORR.
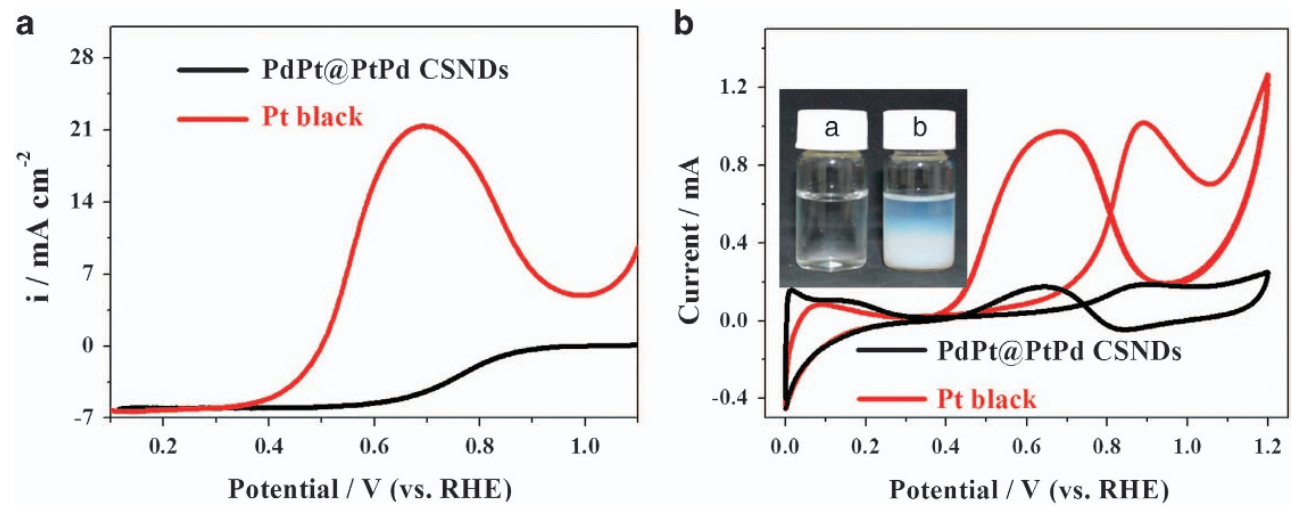

Figure 4 (a) Oxygen reduction reaction (ORR) polarization curves of PdPt@PtPd CSNDs and Pt black in $\mathrm{O}_{2}$-saturated $0.5 \mathrm{M} \mathrm{H}_{2} \mathrm{SO}_{4}$ electrolyte in the presence of $0.1 \mathrm{M} \mathrm{CH}_{3} \mathrm{CH}_{2} \mathrm{OH}$ at $5 \mathrm{mV} \mathrm{s}^{-1}$ and rotation rate of 1600 r.p.m. (b) Electrochemically active surface area (ECSA)-normalized cyclic voltammograms of PdPt@PtPd CSNDs and Pt black in $\mathrm{N}_{2}$-saturated $0.5 \mathrm{M} \mathrm{H}_{2} \mathrm{SO}_{4}+0.1 \mathrm{M} \mathrm{CH}_{3} \mathrm{CH}_{2} \mathrm{OH}$ electrolyte at $50 \mathrm{mV} \mathrm{s}^{-1}$. Insert indicates the digital photographs of (a) $1 \mathrm{ml}$ of $0.5 \mathrm{~m}$ polyallylamine (PAH) aqueous solution after the addition of (a) $5 \mathrm{ml}$ water and (b) $5 \mathrm{ml}$ ethanol. As observed, white flocculation precipitation appears immediately after the addition of ethanol, indicating PAH is ethanol phobic. 
The electrochemically active surface area (ECSA) of PdPt@PtPd CSNDs and commercial Pt black are calculated to be 27.4 and $17.1 \mathrm{~m}^{2} \mathrm{~g}^{-1}$ using a hydrogen adsorption-desorption method. ${ }^{22,33,34}$ Meanwhile, PdPt@PtPd CSNDs show a slight positive shift in the reduction peak potential of surface oxide compared with Pt black, indicating the $\mathrm{PAH}$ functionalization molecule blocks surface oxidation of Pt to some extent because of the interaction between $\mathrm{PAH}$ and Pt. ${ }^{19}$ After CV experiments, the $\mathrm{N}$ 1s peak is still distinctly observed for PdPt@PtPd CSNDs (Supplementary Figure S9), indicating PAH molecules still adsorb on the surface of PdPt@PtPd CSNDs.

The electrocatalytic performances of PdPt@PtPd CSNDs and Pt black were investigated by a $\mathrm{RDE}$ technique in an $\mathrm{O}_{2}$-saturated $0.5 \mathrm{M}$ $\mathrm{H}_{2} \mathrm{SO}_{4}$ electrolyte (Figure $3 \mathrm{~b}$ ). The current densities were normalized to the geometrical area of the electrode. Onset potential of ORR $\left(E_{\mathrm{ORR}}\right.$, a potential with $-10 \mu \mathrm{A} \mathrm{cm}^{-2}$ current $^{35,36}$ ) and half-wave potential of ORR $\left(E_{1 / 2}\right.$, a potential at midpoint of residual current and limited diffusion current) on PdPt@PtPd CSNDs show a $30 \mathrm{mV}$ (1.09 vs $1.06 \mathrm{~V}$ ) and $30 \mathrm{mV}(0.84$ vs $0.81 \mathrm{~V})$ positive shift compared with $\mathrm{Pt}$ black, showing the enhanced ORR kinetics for PdPt@PtPd CSNDs. The area-specific kinetic current density $\left(i_{k}\right)$, an important indicator of electrocatalytic activity, can be calculated by the Koutecky-Levich equation and corresponding ORR polarization curve. ${ }^{19,30}$ As observed, $i_{k}$ of PdPt@PtPd CSNDs $\left(1.60 \mathrm{~mA} \mathrm{~cm}^{-2}\right)$ is 1.80 times bigger than that of Pt black $\left(0.89 \mathrm{~mA} \mathrm{~cm}^{-2}\right)$ at $0.9 \mathrm{~V}$ (insert in Figure $2 \mathrm{~b}$ ), and this is also bigger than some previous values such as $\mathrm{Pt}-\mathrm{Cu}$ bimetallic catalysts $\left(0.84 \mathrm{~mA} \mathrm{~cm}^{-2}\right),{ }^{37}$ net-like Pt assembly $\left(0.688 \mathrm{~mA} \mathrm{~cm}^{-2}\right),{ }^{38}$ Pt-on-Pd nanostructures $\left(0.67 \mathrm{~mA} \mathrm{~cm}^{-2}\right)^{23}$ and $\mathrm{Pt} @ \mathrm{PdCu}_{5} / \mathrm{C}$

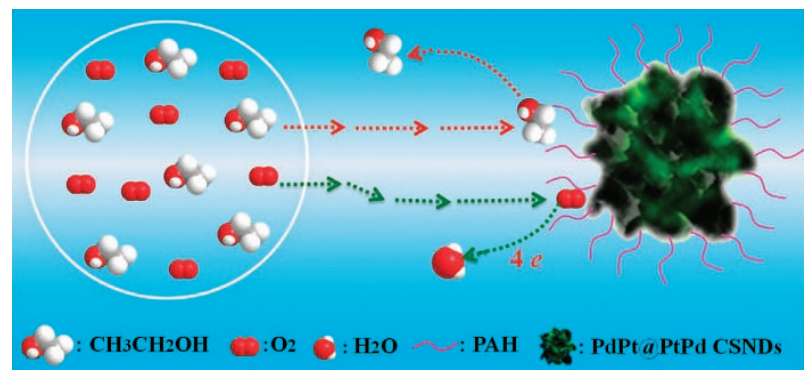

Scheme 2 Schematic illustration of the differential diffusion and reaction of ethanol and $\mathrm{O}_{2}$ on the polyallylamine (PAH)-functionalized PdPt@PtPd CSNDs surface. catalyst $\left(0.543 \mathrm{~mA} \mathrm{~cm}^{-2}\right) .{ }^{39}$ The electronic effect induced by $\mathrm{PAH}$ functionalization, ${ }^{30}$ the increase in interfacial $\mathrm{H}^{+}$concentration induced by PAH functionalization ${ }^{19}$ and the synergistic effect between Pt and Pd elements ${ }^{40}$ (Supplementary Figure S10) are responsible for the improved ORR activity. Furthermore, the ORR activities of electrocatalysts were also normalized to the ECSA and Pt mass of electrocatalysts. The ECSA-specific activity $\left(0.464 \mathrm{~A} \mathrm{~m}^{-2}\right)$ and Pt-mass activity $\left(21.11 \mathrm{~A} \mathrm{~g}^{-1} \mathrm{Pt}\right)$ of PdPt@PtPd CSNDs for the ORR are 1.21 and 3.28 times bigger than that of Pt black $\left(0.384 \mathrm{~A} \mathrm{~m}^{-2}\right.$ and $\left.6.43 \mathrm{~A} \mathrm{~g}^{-1} \mathrm{Pt}\right)$ at $0.9 \mathrm{~V}$ respectively (Supplementary Figure S11), indicating PdPt@PtPd CSNDs hold promise as a potentially practical cathodic ORR electrocatalyst.

The ethanol tolerance of PdPt@PtPd CSNDs and Pt black were investigated by RDE and $\mathrm{CV}$ tests in the presence of ethanol. A large anodic current of ethanol oxidation is observed at the ORR polarization curve of Pt black but no anodic current is observed at the ORR polarization curve of PdPt@PtPd CSNDs (Figure 4a). Compared with Pt black, PdPt@PtPd CSNDs show a $517 \mathrm{mV}$ increase in $\mathrm{E}_{\mathrm{ORR}}$, demonstrating their high ORR activity in the presence of ethanol (Figure $4 \mathrm{~b}$ ). Further CV tests show ECSA-normalized specific peak current density of ethanol oxidation for PdPt@PtPd CSNDs is 8.7 times lower than that for Pt black (Figure 3d). Meanwhile, CV and $\mathrm{RDE}$ tests show the unfunctionalized PtPd alloy nanoparticles have not obvious alcohol tolerance for the ORR (Supplementary Figure S12), indicating the ethanol tolerance of PdPt@PtPd CSNDs cannot be ascribed to alloying effect. Thus, the present experimental results indicate the PAH on the PdPt@PtPd CSNDs surface serves effectively as barrier networks to block accessibility of ethanol, owing to its lager molecular size (size: $5.1 \AA$ ) than the $\mathrm{O}_{2}$ molecule (size: $3.4 \AA$ ), as shown in Scheme 2. To confirm molecular size-dependent selectivity, the CO stripping, methanol oxidation reaction and 1-propanol oxidation reaction on catalysts were further investigated by $\mathrm{CV}$. COstripping measurements show the oxidation charge of pre-absorbed CO on PdPt@PtPd CSNDs is higher than that on commercial Pt black (Supplementary Figure S13), confirming ECSA of PdPt@PtPd CSNDs is bigger than that of commercial Pt black. Meanwhile, it is observed that specific activity of PdPt@PtPd CSNDs decreases with increasing molecule size of alcohol, relative to commercial Pt black (Supplementary Figure S14), confirming the low ethanol oxidation activity on PdPt@PtPd CSNDs is due to the molecular size sorting by $\mathrm{PAH}$. Moreover, the solubility experiments demonstrate $\mathrm{PAH}$ is ethanol phobic (insert in Figure 4b). Thus, PAH on PdPt@PtPd

b

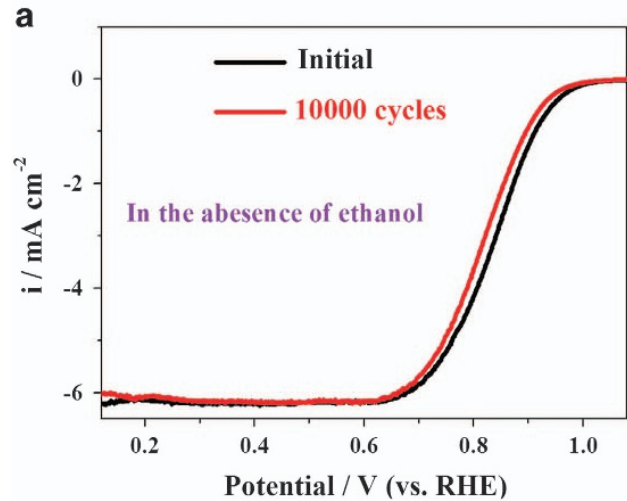

Figure 5 Oxygen reduction reaction (ORR) polarization curves of PdPt@PtPd CSNDs before and after 10000 potential sweeps in $\mathrm{O}_{2}$-saturated $0.5 \mathrm{M} \mathrm{H}_{2} \mathrm{SO}_{4}$ electrolyte in (a) the absence and (b) presence of $0.1 \mathrm{M} \mathrm{CH}_{3} \mathrm{CH}_{2} \mathrm{OH}$ at $5 \mathrm{mV} \mathrm{s}^{-1}$ and rotation rate of 1600 r.p.m. 
CSNDs surface also blocks ethanol diffusion from aqueous solution to the active Pt sites.

The accelerated durability test ${ }^{41}$ (that is, continuously applying linear potential sweep between 0.6 and $1.1 \mathrm{~V}$ vs reversible hydrogen electrode in $\mathrm{O}_{2}$-saturated $0.5 \mathrm{M} \mathrm{H}_{2} \mathrm{SO}_{4}$ electrolyte at $50 \mathrm{mV} \mathrm{s}^{-1}$ ) was performed to investigate the electrocatalytic stability and long-term selectivity (that is, ethanol tolerance) of PdPt@PtPd CSNDs and Pt black. After 10000 potential sweeps, PdPt@PtPd CSNDs have a $22 \mathrm{mV}$ degradation in $E_{1 / 2}$ for the ORR (Figure $5 \mathrm{a}$ ), whereas Pt black has a $55 \mathrm{mV}$ degradation in $E_{1 / 2}$ for the ORR (Supplementary Figure S15). Although the chemical stability of the Pd element (that is, the dissolution in acidic media) is lower than that of the Pt element, the present experimental results demonstrate that PdPt@PtPd CSNDs have higher electrocatalytic stability than commercial Pt black. The particular 3D self-supported architecture that effectively restrains the Ostwald ripening effect ${ }^{42-44}$ contributes to the enhanced stability of PdPt@PtPd CSNDs (Supplementary Figure S16). The additional accelerated durability test measurements show the ORR stability of PdPt@PtPd CSNDs is close to that of as-prepared Pt nanodendrites and much higher than that of as-prepared Pd nanodendrites (Supplementary Figure S17), and this can be ascribed to high $\mathrm{Pt}$ content on the PtPd alloy shell of PdPt@PtPd CSNDs. In addition, very importantly, the ethanol tolerance of PdPt@PtPd CSNDs is still retained well after 10000 cycles (Figure 5b), clearly suggesting their long-term selectivity for the ORR. After the accelerated durability test experiments, the $\mathrm{N}$ 1s peak of PAH is still detected (Supplementary Figure S18). Thus, the high stability of the $\mathrm{PAH}$ functionalization molecules contributes to the long-term selectivity of PdPt@PtPd CSNDs for the ORR.

\section{CONCLUSIONS}

In summary, for the first time, we reported a facile water-based thermal decomposition approach to synthesize the well-defined chemical functionalized PdPt@PtPd CSNDs by using a simultaneously $\mathrm{PAH}$ complexant, reductant and chemical functionalized molecule that could be used directly as an ORR electrocatalyst without the tedious UV/ozone post-treatment procedure. The PAH-functionalized PdPt@PtPd CSNDs showed an enhanced ORR activity and durability compared with commercial Pt black because of the increased interfacial $\mathrm{H}^{+}$concentration, electronic effect, synergistic effect and particular 3D self-supported architecture. Meanwhile, the PAH functionalization imparted PdPt@PtPd CSNDs with extraordinary selectivity for the ORR because of its steric hindrance effect and ethanol-phobic property, making them indeed promising cathodic electrocatalysts for the practical DAFC application.

\section{CONFLICT OF INTEREST}

The authors declare no conflict of interest.

\section{ACKNOWLEDGEMENTS}

This work was supported by National Natural Science Foundation of China (21473111 and 21301114), Natural Science Foundation of Shaanxi Province (2015JM2043 and 2013JQ2009), Fundamental Research Funds for the Central Universities (GK201402016) and the Academic Research Fund of the Ministry of Education in Singapore (RGT27/13).

1 Wu, Z., Lv, Y., Xia, Y., Webley, P. A. \& Zhao, D. Ordered mesoporous platinum@graphitic carbon embedded nanophase as a highly active, stable, and methanol-tolerant oxygen reduction electrocatalyst. J. Am. Chem. Soc. 134, 2236-2245 (2011).
2 Chang, J., Feng, L., Liu, C., Xing, W. \& Hu, X. Ni 2 P enhances the activity and durability of the Pt anode catalyst in direct methanol fuel cells. Energy Environ. Sci. 7 , 1628-1632 (2014)

3 Tiwari, J. N., Tiwari, R. N., Singh, G. \& Kim, K. S. Recent progress in the development of anode and cathode catalysts for direct methanol fuel cells. Nano Energy 2 553-578 (2013).

4 Xu, H., Ding, L.-X., Liang, C.-L., Tong, Y.-X. \& Li, G.-R. High-performance polypyrrole functionalized PtPd electrocatalysts based on PtPd/PPy/PtPd three-layered nanotube arrays for the electrooxidation of small organic molecules. NPG Asia Mater. 5 e69 (2013)

5 Zhang, K., Yang, W., Ma, C., Wang, Y., Sun, C. W., Chen, Y. J., Duchesne, P., Zhou, J. G., Wang, J., Hu, Y. F., Banis, M. N., Zhang, P., Li, F., Li, J. G. \& Chen, L. Q. A highly active, stable and synergistic Pt nanoparticles/ $\mathrm{Mo}_{2} \mathrm{C}$ nanotube catalyst for methanol electro-oxidation. NPG Asia Mater. 7, e153 (2015).

6 Tan, Y. M., Xu, C. F., Chen, G. X., Zheng, N. F. \& Xie, Q. J. A graphene-platinum nanoparticles-ionic liquid composite catalyst for methanol-tolerant oxygen reduction reaction. Energy Environ. Sci. 5, 6923-6927 (2012).

7 Zhao, X., Yin, M., Ma, L., Liang, L., Liu, C. P., Liao, J. H., Lu, T. H. \& Xing, W. Recent advances in catalysts for direct methanol fuel cells. Energy Environ. Sci. 4 2736-2753 (2011).

8 Yu, T., Kim, D. Y., Zhang, H. \& Xia, Y. Platinum concave nanocubes with high-index facets and their enhanced activity for oxygen reduction reaction. Angew. Chem. Int. Ed. 50, 2773-2777 (2011)

9 Xu, X., Zhang, X., Sun, H., Yang, Y., Dai, X., Gao, J., Li, X., Zhang, P., Wang, H. H., Yu, N. F. \& Sun, S. G. Synthesis of Pt-Ni alloy nanocrystals with high-index facets and enhanced electrocatalytic properties. Angew. Chem. 126, 12730-12735 (2014)

10 Shui, J. I., Chen, C. \& Li, J. C. M. Evolution of nanoporous Pt-Fe alloy nanowires by dealloying and their catalytic property for oxygen reduction reaction. Adv. Funct. Mater. 21, 3357-3362 (2011).

11 Carpenter, M. K., Moylan, T. E., Kukreja, R. S., Atwan, M. H. \& Tessema, M. M. Solvothermal synthesis of platinum alloy nanoparticles for oxygen reduction electrocatalysis. J. Am. Chem. Soc. 134, 8535-8542 (2012).

12 Ge, X. B., Chen, L. y., Kang, J. L., Fujita, T., Hirata, A., Zhang, W., Jiang, J. H. \& Chen, M. W. A core-shell nanoporous Pt-Cu catalyst with tunable composition and high catalytic activity. Adv. Funct. Mater. 23, 4156-4162 (2013).

13 Lang, X. Y., Han, G. F., Xiao, B. B., Gu, L., Yang, Z. Z., Wen, Z., Zhu, Y. F., Zhao, M., $\mathrm{Li}$, J. C. \& Jiang, Q. Mesostructured intermetallic compounds of platinum and nontransition metals for enhanced electrocatalysis of oxygen reduction reaction. Adv. Funct. Mater. 25, 230-237 (2015).

$14 \mathrm{He}, \mathrm{C}$. \& Shen, P. K. Pt loaded on truncated hexagonal pyramid WC/graphene for oxygen reduction reaction. Nano Energy 8, 52-61 (2014).

15 Kok Poh, C., Lim, S. H., Tian, Z. Q., Lai, L. F., Feng, Y. P., Shen, Z. X. \& Lin, J. Y. $\mathrm{Pt}-\mathrm{W}_{\mathrm{x}} \mathrm{C}$ nano-composites as an efficient electrochemical catalyst for oxygen reduction reaction. Nano Energy 2, 28-39 (2013).

16 Kibsgaard, J., Gorlin, Y., Chen, Z. \& Jaramillo, T. F. Meso-structured platinum thin films: active and stable electrocatalysts for the oxygen reduction reaction. J. Am. Chem. Soc. 134, 7758-7765 (2012)

17 Xia, B. Y., Ng, W. T., Wu, H. B., Wang, X. \& Lou, X. W. D. Self-supported interconnected Pt nanoassemblies as highly stable electrocatalysts for lowtemperature fuel cells. Angew. Chem. 124, 7325-7328 (2012).

18 Strmcnik, D., Escudero-Escribano, M., Kodama, K., Stamenkovic, V. R., Cuesta, A. \& Marković, N. M. Enhanced electrocatalysis of the oxygen reduction reaction based on patterning of platinum surfaces with cyanide. Nat. Chem. 2 880-885 771 (2010)

19 Fu, G., Jiang, X., Gong, M., Chen, Y., Tang, Y., Lin, J. \& Lu, T. Highly branched platinum nanolance assemblies by polyallylamine functionalization as superior active, stable, and alcohol-tolerant oxygen reduction electrocatalysts. Nanoscale 6 , 8226-8234 (2014)

$20 \mathrm{Lu}$, Y., Jiang, Y. \& Chen, W. PtPd porous nanorods with enhanced electrocatalytic activity and durability for oxygen reduction reaction. Nano Energy 2, 836-844 (2013).

21 Koenigsmann, C., Santulli, A. C., Gong, K., Vukmirovic, M. B., Zhou, W. P., Sutter, E., Wong, S. S. \& Adzic, R. R. Enhanced electrocatalytic performance of processed, ultrathin, supported Pd-Pt core-shell nanowire catalysts for the oxygen reduction reaction. J. Am. Chem. Soc. 133, 9783-9795 (2011).

22 Hong, J. W., Kang, S. W., Choi, B. S., Kim, D., Lee, S. B. \& Han, S. W. Controlled synthesis of Pd-Pt alloy hollow nanostructures with enhanced catalytic activities for oxygen reduction. ACS Nano 6, 2410-2419 (2012)

23 Peng, Z. \& Yang, H. Synthesis and oxygen reduction electrocatalytic property of Pt-on-Pd bimetallic heteronanostructures. J. Am. Chem. Soc. 131, $7542-7543$ (2009)

24 Genorio, B., Strmcnik, D., Subbaraman, R., Tripkovic, D., Karapetrov, G. Stamenkovic, V. R., Pejovnik, S. \& Marković, N. M. Selective catalysts for the hydrogen oxidation and oxygen reduction reactions by patterning of platinum with calix 4 arene molecules. Nat. Mater. 9, 998-1003 (2010).

25 Genorio, B., Subbaraman, R., Strmcnik, D., Tripkovic, D., Stamenkovic, V. R. \& Marković, N. M. Tailoring the selectivity and stability of chemically modified platinum nanocatalysts to design highly durable anodes for PEM fuel cells. Angew. Chem. Int Edit. 50, 5468-5472 (2011)

26 Liu, H., Qu, J., Chen, Y., Li, J., Ye, F., Lee, J. Y. \& Yang, J. Hollow and cage-bell structured nanomaterials of noble metals. J. Am. Chem. Soc. 134 11602-11610 (2012). 
27 Garsany, Y., Baturina, O. A., Swider-Lyons, K. E. \& Kocha, S. S. Experimental methods for quantifying the activity of platinum electrocatalysts for the oxygen reduction reaction. Anal. Chem. 82, 6321-6328 (2010).

28 Liu, P., Zhai, M., Li, J., Peng, J. \& Wu, J. Radiation preparation and swelling behavior of sodium carboxymethyl cellulose hydrogels. Radiat. Phys. Chem. 63, 525-528 (2002).

29 Taniguchi, A. \& Cakmak, M. The suppression of strain induced crystallization in PET through sub micron $\mathrm{TiO}_{2}$ particle incorporation. Polymer 45, 6647-6654 (2004).

30 Fu, G., Wu, K., Jiang, X., Tao, L., Chen, Y., Lin, J., Zhou, Y., Wei, S., Tang, Y., Lu, T. \& $\mathrm{Xia}, \mathrm{X}$. Polyallylamine-directed green synthesis of platinum nanocubes. Shape and electronic effect codependent enhanced electrocatalytic activity. Phys. Chem. Chem. Phys. 15, 3793-3802 (2013).

31 Fu, G. T., Han, W, Yao, L. F., Lin, J, Wei, S. H., Chen, Y, Tang, Y. W., Zhou, Y. M., Lu, T. H. \& Xia, X. H. One-step synthesis and catalytic properties of porous palladium nanospheres. J. Mater. Chem. 22, 17604-17611 (2012).

32 Moulder, J., Stickle, W., Sobol, P. \& Bomben, K. Handbook of X-Ray Photoelectron Spectroscopy(Perkin-Elmer Corporation, Physical Electronics Division: Eden Prairie, MN, (1992).

33 Yuan, Q., Zhou, Z., Zhuang, J. \& Wang, X. Pd-Pt random alloy nanocubes with tunable compositions and their enhanced electrocatalytic activities. Chem. Commun. 46, 1491-1493 (2010).

34 Ren, F., Wang, H., Zhai, C., Zhu, M., Yue, R., Du, Y., Yang, P., Xu, J. \& Lu, W. Clean method for the synthesis of reduced graphene oxide-supported PtPd alloys with high electrocatalytic activity for ethanol oxidation in alkaline medium. ACS Appl. Mater. Inter. 6, 3607-3614 (2014).

35 Kannari, N. \& Ozaki, J.-i. Formation of uniformly and finely dispersed nanoshells by carbonization of cobalt-coordinated oxine-formaldehyde resin and their electrochemical oxygen reduction activity. Carbon 50, 2941-2952 (2012).

36 Niwa, H., Horiba, K., Harada, Y., Oshima, M., Ikeda, T., Terakura, K., Ozaki, J.-i. \& Miyata, S. X-ray absorption analysis of nitrogen contribution to oxygen reduction reaction in carbon alloy cathode catalysts for polymer electrolyte fuel cells. J. Power Sources 187, 93-97 (2009).

37 Zhu, C.-M., Gao, A., Wang, Y. \& Liu, Y. Pt-Cu bimetallic electrocatalysts with enhanced catalytic properties for oxygen reduction. Chem. Commun. 50, 13889-13892 (2014).
38 Wang, H. H., Zhou, Z. Y., Yuan, Q. A., Tian, N. \& Sun, S. G. Pt nanoparticle netlikeassembly as highly durable and highly active electrocatalyst for oxygen reduction reaction. Chem. Commun. 47, 3407-3409 (2011).

39 Cochell, T. \& Manthiram, A. Pt@Pdx $\mathrm{Cu}_{y} / \mathrm{C}$ core-shell electrocatalysts for oxygen reduction reaction in fuel cells. Langmuir 28, 1579-1587 (2012)

40 Kang, Y., Ye, X., Chen, J., Cai, Y., Diaz, R. E., Adzic, R. R., Stach, E. A. \& Murray, C. B. Design of Pt-Pd binary superlattices exploiting shape effects and synergistic effects for oxygen reduction reactions. J. Am. Chem. Soc. 135, 42-45 (2012).

41 Lim, B., Jiang, M., Camargo, P. H., Cho, E. C., Tao, J., Lu, X., Zhu, Y. \& Xia, Y. Pd-Pt bimetallic nanodendrites with high activity for oxygen reduction. Science 324 1302-1305 1170377 (2009)

42 Xia, B. Y., Ng, W. T., Bin Wu, H., Wang, X. \& Lou, X. W. Self-supported interconnected Pt nanoassemblies as highly stable electrocatalysts for low-temperature fuel cells. Angew. Chem. Int. Edit. 51, 7213-7216 (2012).

43 Sun, S. H., Zhang, G., Geng, D., Chen, Y., Li, R., Cai, M. \& Sun, X. A highly durable platinum nanocatalyst for proton exchange membrane fuel cells: multiarmed starlike nanowire single crystal. Angew. Chem. Int. Edit. 50, 422-426 (2011).

44 Mourdikoudis, S., Chirea, M., Altantzis, T., Pastoriza-Santos, I., Pérez-Juste, J., Silva, F., Bals, S. \& Liz-Marzán, L. M. Dimethylformamide-mediated synthesis of water-soluble platinum nanodendrites for ethanol oxidation electrocatalysis. Nanoscale 5, 4776-4784 (2013).

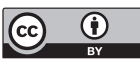

This work is licensed under a Creative Commons Attribution 4.0 International License. The images or other third party material in this article are included in the article's Creative Commons license, unless indicated otherwise in the credit line; if the material is not included under the Creative Commons license, users will need to obtain permission from the license holder to reproduce the material. To view a copy of this license, visit http:// creativecommons.org/licenses/by/4.0/

Supplementary Information accompanies the paper on the NPG Asia Materials website (http://www.nature.com/am) 